\title{
Summary Statistics for Fun Dough Data Acquired at LLNL
}

Jeffrey S. Kallman, Kenneth E. Morales, Richard E. Whipple, Robert D. Huber, William D. Brown, Jerel A. Smith, Daniel J. Schneberk, Harry E. Martz Jr., W.

Travis White III

Lawrence Livermore National Laboratory

Livermore, CA 94551

Work performed on the

Science \& Technology Directorate of the

Department of Homeland Security

Statement of Work

PR\# RSEN-10-00023

IAA HSHQPM-10-X-00005

3/12/2010

LLNL-TR-425703

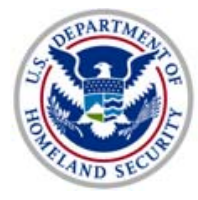


This document was prepared as an account of work sponsored by an agency of the United States government. Neither the United States government nor Lawrence Livermore National Security, LLC, nor any of their employees makes any warranty, expressed or implied, or assumes any legal liability or responsibility for the accuracy, completeness, or usefulness of any information, apparatus, product, or process disclosed, or represents that its use would not infringe privately owned rights. Reference herein to any specific commercial product, process, or service by trade name, trademark, manufacturer, or otherwise does not necessarily constitute or imply its endorsement, recommendation, or favoring by the United States government or Lawrence Livermore National Security, LLC. The views and opinions of authors expressed herein do not necessarily state or reflect those of the United States government or Lawrence Livermore National Security, LLC, and shall not be used for advertising or product endorsement purposes.

This work performed under the auspices of the U.S. Department of Energy by Lawrence Livermore National Laboratory under Contract DE-AC52-07NA27344. 


\section{Executive Summary}

\begin{tabular}{|c|c|c|c|c|c|}
\hline \multicolumn{2}{|c|}{ Measured Density: $1.2 \mathrm{~g} / \mathrm{cm}^{3}$} & \multicolumn{4}{|c|}{ X-ray beam energy and source filter material(s) } \\
\hline \multicolumn{2}{|r|}{ Parameter } & $100 \mathrm{kVp}(\mathrm{Al})$ & $160 \mathrm{kVp}(\mathrm{Al})$ & $160 \mathrm{kVp}(\mathrm{AlCu})$ & $300 \mathrm{kVp}(\mathrm{Cu})$ \\
\hline \multirow{5}{*}{$L A C$} & Mean Measured LAC $\left(L M H U_{D}\right)$ & 2075 & 1830 & 1288 & 1121 \\
\hline & Standard Deviation (\% of Mean) & $3 \%$ & $4 \%$ & $4 \%$ & $5 \%$ \\
\hline & Entropy & 5.52 & 5.58 & 5.41 & 5.54 \\
\hline & Modeled LAC $\left(L M H U_{D}\right)$ & (na) & (na) & (na) & (na) \\
\hline & Difference Between Model \& Mean & (na) & (na) & (na) & (na) \\
\hline \multirow{2}{*}{$Z_{\text {eff }}$} & From the mean measured LAC & \multicolumn{4}{|l|}{8.6} \\
\hline & From the modeled LAC & \multicolumn{4}{|l|}{ (na) } \\
\hline
\end{tabular}

Table 1. First-order statistics of the $x$-ray linear attenuation coefficient (LAC) in Fun Dough ${ }^{\mathrm{TM}}$ and the estimated value of $Z_{\text {eff- }}$

Using x-ray computerized tomography (CT), we have characterized the x-ray linear attenuation coefficients (LAC) of a Play Dough ${ }^{\mathrm{TM}}$-like product, Fun Dough ${ }^{\mathrm{TM}}$, designated as PD. [1, 2] Table 1 gives the first-order statistics for each of four CT measurements, estimated with a Gaussian kernel density estimator (KDE) analysis. [3]. The mean values of the LAC range from a high of about $2100 \mathrm{LMHU}_{\mathrm{D}}{ }^{1}$ at $100 \mathrm{kVp}$ to a low of about $1100 \mathrm{LMHU}_{\mathrm{D}}$ at $300 \mathrm{kVp}$. The standard deviation of each measurement is around $1 \%$ of the mean. The entropy covers the range from 3.9 to 4.6. Ordinarily, we would model the LAC of the material and compare the modeled values to the measured values. In this case, however, we did not have the composition of the material and therefore did not model the LAC. Using a method recently proposed by Lawrence Livermore National Laboratory (LLNL), [4] we estimate the value of the

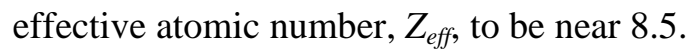

LLNL prepared about 50mL of the Fun Dough ${ }^{\mathrm{TM}}$ in a polypropylene vial and firmly compressed it immediately prior to the x-ray measurements. Still, layers can plainly be seen in the reconstructed images, indicating that the bulk density of the material in the container is affected by voids and bubbles. We used the computer program IMGREC to reconstruct the CT images. The values of the key parameters used in the data capture and image reconstruction are given in this report. Additional details may be found in the experimental SOP [1] and a separate document.[2] To characterize the statistical distribution of LAC values in each CT image, we first isolated an $80 \%$ central-core segment of volume elements ("voxels") lying completely within the specimen, away from the walls of the polypropylene vial. All of the voxels within this central core, including those comprised of voids and inclusions, are included in the statistics. We then calculated the mean value, standard deviation and entropy for (a) the four image segments and for (b) their digital gradient images. (A digital gradient image of a given image was obtained by taking the absolute value of the difference between the initial image and that same image offset by one voxel horizontally, parallel to the rows of the x-ray detector array.) The statistics of the initial image of LAC values are called "first order statistics;" those of the gradient image, "second order statistics."

\footnotetext{
${ }^{1} \mathrm{LMHU}_{\mathrm{D}}$ : “LLNL $\underline{\text { modified }}$ Hounsfield units with respect to Delrin.” To obtain the LAC in $\mathrm{LMHU}_{\mathrm{D}}$ for some material at a specific energy, one divides the LAC of that material (in units of reciprocal $\mathrm{mm}$ ) for that energy by the LAC of Delrin for $160 \mathrm{kVp}$ (also in reciprocal $\mathrm{mm}$ ) and multiplies the result by 1400 . The result is a dimensionless value. The LAC of Delrin used to normalize all LAC values in this report is the value measured with an $\mathrm{x}$-ray energy of $160 \mathrm{kV}$ with combined aluminum and copper filters.
} 
Summary of PD (Fun Dough) X-ray Statistics

Report Date: $3 / 11 / 2010$

Author: W. Travis White, III

Typed or Printed Name

LLNL

Organization

QA: _ Robert D. Huber

Typed or Printed Name

$\frac{\text { LLNL }}{\text { Organization }}$

Material ID(s):

Fun Dough TM

\begin{tabular}{|c|c|c|c|c|c|c|c|c|}
\hline \multicolumn{3}{|c|}{ Source } & \multirow{2}{*}{$\begin{array}{c}\text { Collimator } \\
\begin{array}{c}\text { Number of } \\
\text { slits }\end{array}\end{array}$} & \multirow{2}{*}{$\begin{array}{c}\begin{array}{c}\text { Sample } \\
\text { Preparation }\end{array} \\
\text { Date }\end{array}$} & \multirow{2}{*}{$\begin{array}{c}\begin{array}{c}\text { X-ray } \\
\text { Measurement }\end{array} \\
\text { Date }\end{array}$} & \multicolumn{3}{|c|}{$\begin{array}{c}\text { Linear Attenuation Coefficient } \\
\text { (LAC) }\end{array}$} \\
\hline $\begin{array}{l}\text { Bias } \\
(\mathbf{k V})\end{array}$ & Mate & $\begin{array}{l}\text { Iters } \\
\text { Thickness }\end{array}$ & & & & Statistic & $1^{\text {st }}$ order & $\begin{array}{c}2^{\text {nd }} \\
\text { order }\end{array}$ \\
\hline 100 & $\mathrm{Al}$ & $1.943 \mathrm{~mm}$ & 2 & 02/18/2010 & 02/18/2010 & $\begin{array}{c}\text { Mean } \\
\text { Std. Dev. } \\
\text { Entropy } \\
\text { Calculated }\end{array}$ & $\begin{array}{r}2075 \\
62 \\
5.52 \\
\text { (na) }\end{array}$ & $\begin{array}{r}48 \\
37 \\
4.85 \\
\text { (na) } \\
\end{array}$ \\
\hline 160 & $\mathrm{Al}$ & $1.943 \mathrm{~mm}$ & 2 & 02/18/2010 & 02/18/2010 & $\begin{array}{c}\text { Mean } \\
\text { Std. Dev. } \\
\text { Entropy } \\
\text { Calculated }\end{array}$ & $\begin{array}{r}1830 \\
64 \\
5.58 \\
\text { (na) }\end{array}$ & $\begin{array}{r}52 \\
40 \\
4.94 \\
\text { (na) }\end{array}$ \\
\hline 160 & $\begin{array}{l}\mathrm{Al} \\
\mathrm{Cu}\end{array}$ & $\begin{array}{l}1.943 \mathrm{~mm} \\
1.905 \mathrm{~mm}\end{array}$ & 2 & 02/18/2010 & 02/18/2010 & $\begin{array}{c}\text { Mean } \\
\text { Std. Dev. } \\
\text { Entropy } \\
\text { Calculated }\end{array}$ & $\begin{array}{r}1288 \\
54 \\
5.41 \\
\text { (na) }\end{array}$ & $\begin{array}{r}48 \\
37 \\
4.86 \\
\text { (na) }\end{array}$ \\
\hline 300 & $\mathrm{Cu}$ & $2.972 \mathrm{~mm}$ & 2 & 02/18/2010 & 02/18/2010 & $\begin{array}{c}\text { Mean } \\
\text { Std. Dev. } \\
\text { Entropy } \\
\text { Calculated }\end{array}$ & $\begin{array}{r}1121 \\
61 \\
5.54 \\
\text { (na) }\end{array}$ & $\begin{array}{r}56 \\
43 \\
5.01 \\
\text { (na) }\end{array}$ \\
\hline $\mathbf{Z}_{\text {eff }}$ & & & & $\mathrm{Z}_{\text {eff }}$ basec & $\frac{Z_{\text {eff }} \text { based on m }}{\text { on calculated (n }}$ & $\frac{\text { asured LAC }}{\text { odeled) LAC }}$ & $\frac{8 .}{(n}$ & \\
\hline
\end{tabular}

Table 2. Key statistics for x-ray measurements of Linear Attenuation Coefficient (LAC).

Comments:

Data Shipped to TSL

TBD

TBD 


\section{SUPPLEMENTAL ANALYSIS}

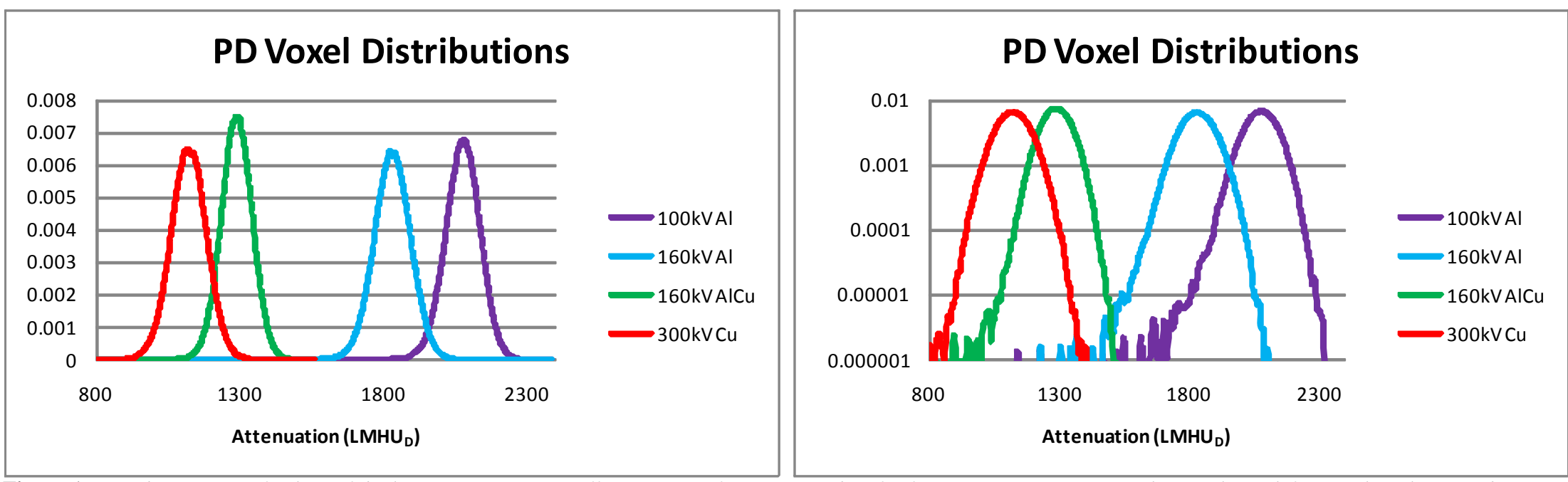

Figure 1. KDE histograms of values of the linear attenuation coefficient (LAC) for Fun Dough ${ }^{\mathrm{TM}}$ for four x-ray source settings (linear plots - left; semi-log plots - right).

Comments/Observations on Histograms: These histograms are made using $150 \mu \mathrm{m}$ voxels

\section{Reference Specimens}

\begin{tabular}{|l|r|r|r|r|r|r|}
\hline & \multicolumn{1}{|l|}{ graphite } & \multicolumn{1}{l|}{ ethanol } & \multicolumn{1}{l|}{ Delrin } & water & Teflon & aluminum \\
\hline \hline $100 \mathrm{kV}(\mathrm{Al})$ Mean & 1830 & 1082 & 1904 & 1486 & 3201 & 7543 \\
$100 \mathrm{kV}(\mathrm{Al})$ Std Dev & 88 & 58 & 86 & 58 & 99 & 136 \\
\hline $160 \mathrm{kV}(\mathrm{AlCu})$ Mean & 1461 & 843 & 1400 & 1046 & 1998 & 3095 \\
$160 \mathrm{kV}(\mathrm{AlCu})$ Std Dev & 60 & 45 & 55 & 46 & 58 & 72 \\
\hline
\end{tabular}

Table 3. Linear attenuation coefficients $\left(L M H U_{D}\right)$ of six reference materials as measured simultaneously with Fun Dough. 


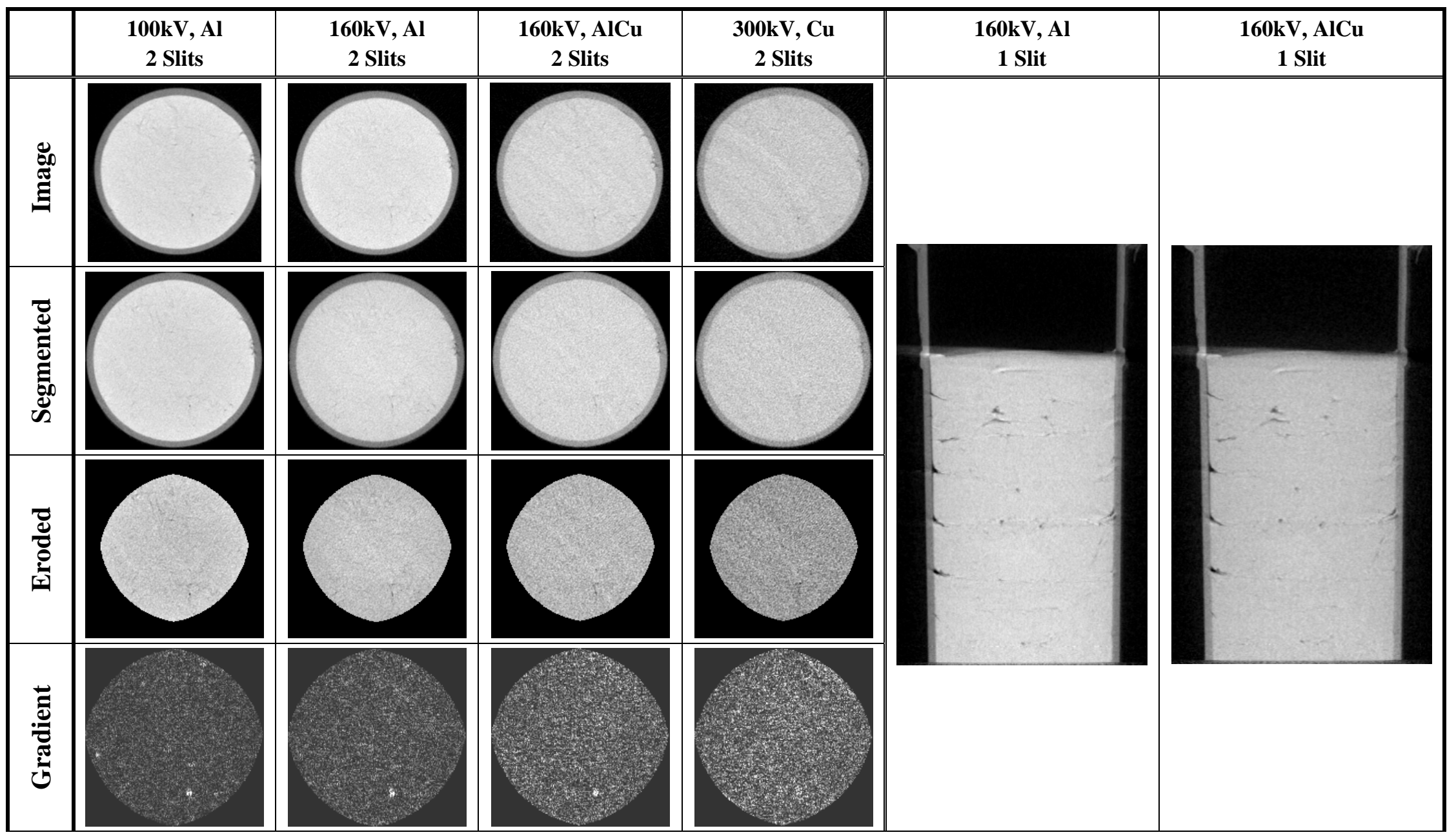

Figure 2. X-ray slice images with $150 \mu \mathrm{m}$ x $150 \mu \mathrm{m}$ x150 $\mu \mathrm{m}$ voxels. Raw data (top row), first-order segmented images (second row), eroded images (third row) used to calculate first order statistics. Fourth row, difference or gradient image used for second-order statistics. Images not to scale.

Comments/Observations on Appearance of Sample (texture, color, other): 


\section{Specimen Preparation}

1. Person responsible:

Rich Whipple

2. Date/time:

$02 / 18 / 2010$

3. Location:

LLNL

4. Identifier:

Fun Dough ${ }^{\mathrm{TM}}$, marketed by Rose Art Industries, Inc., Livingston, NJ 07039

5. Preparation procedure:

Off-the-shelf commercial product

6. Container: Polypropylene vial with attached snap cap, p/n 09-341-75A, Fisher Scientific

7. Nominal Measured Density: ${ }^{1}$ Average density of as- $\mathrm{X}$-rayed specimen $=1.20 \mathrm{~g} / \mathrm{cc}$

8. Image of Sample:

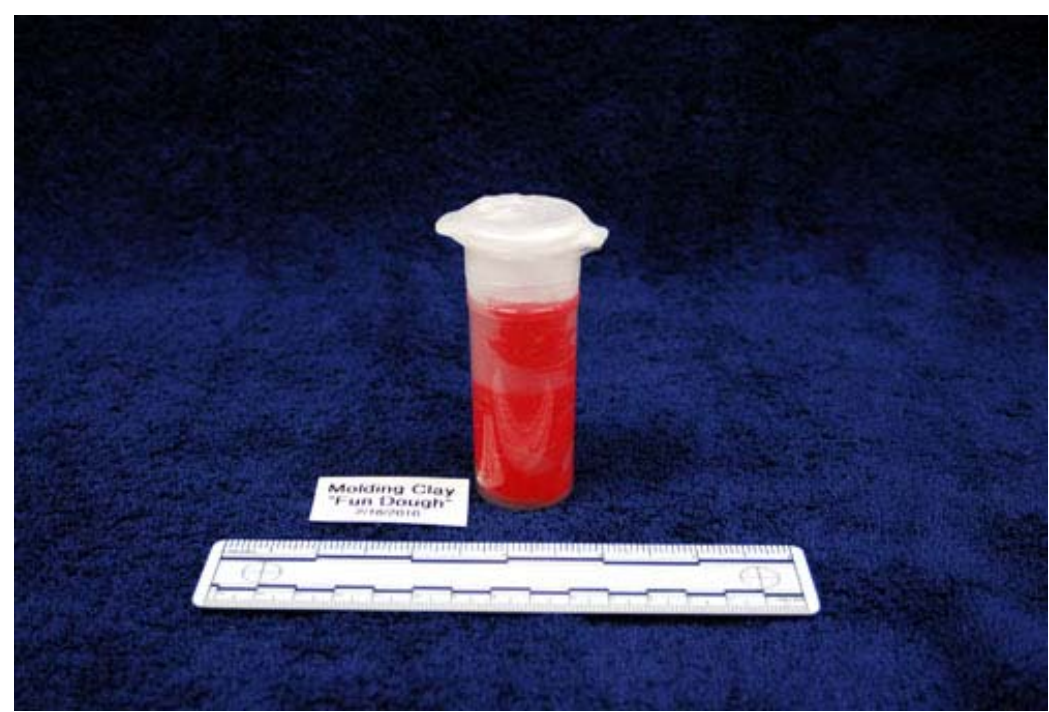

9. Observations:

\footnotetext{
${ }^{1}$ Ratio of mass and volume of the specimen in the container, including cracks and voids.
} 


\section{CT System Configuration}

1. Scan Location Site: LLNL HEAF

2. Source: Yxlon D09 450 kV Tube; Mfr. Catalog Number: 9421-172-33503; S/N 61-0971

3. Detector: Thales Flashscan 33 with Lanex Fine Gadolinium Oxysulfate Scintillator Screen; s/n 392177

4. Rotation control system. Controller: Newport Model ESP7000 SN: 1250

5. Carousel: LLNL 2-tray, 7” Dia.

5. Data capture computer: Dell DHM/J4271

\section{CT Scan Parameters}

1. Scan Geometry: ${ }^{1}$ SOD (mm): $1102 \quad$ ODD (mm): $\underline{286}$

Number of positions: $400 \quad$ Angular Range: $200^{\circ}$

Angular Increment: $\underline{0.5^{\circ}}$

2. Number of Frames averaged per Image: 4

3. Integration time per frame: See $\mathrm{p} 7$.

\footnotetext{
${ }^{1}$ Distances are those recorded in the .sct file for this experiment and are the values used in image reconstruction.
} 


\section{File Storage Locations for X-ray Data}

\section{Specimen}

Root Data Path: /Working /TP35_Industrial_CT_of_HME/LLNL/None/HEAFCAT/None/PD_100218/\{sub directory\}/Raw_Data

\begin{tabular}{|c|c|c|c|c|c|c|c|c|c|c|}
\hline $\begin{array}{c}\text { Specimen } \\
\text { ID }\end{array}$ & Date & Radiographer & Slits & $\mathbf{k V p}$ & $\mathbf{m A}$ & $\begin{array}{c}\text { Al } \\
\text { Filter } \\
(\mathbf{m m})\end{array}$ & $\begin{array}{c}\text { Cu } \\
\text { Filter } \\
(\mathbf{m m})\end{array}$ & $\begin{array}{l}\text { Integration } \\
\text { dpix Setting } \\
\text { [time/frame } \\
\text { (s)] }\end{array}$ & \{sub directory\} & File Name \\
\hline \multirow{6}{*}{ PD } & 100218 & Morales & 2 & 100 & 1.1 & 1.943 & 0 & 10 [3.2s] & /PD_100Al & PD_100Al_nn.sdt ${ }^{1}$ \\
\hline & 100218 & Morales & 2 & 160 & 0.7 & 1.943 & 0 & $5[2.3 \mathrm{~s}]$ & /PD_160Al & PD_160Al_nn.sdt \\
\hline & 100218 & Morales & 2 & 160 & 4.35 & 1.943 & 1.905 & $8[2.8 \mathrm{~s}]$ & /PD_160AlCu & PD_160AlCu_nn.sdt \\
\hline & 100218 & Morales & 2 & 300 & 2.3 & 0 & 2.972 & $4[2.1 \mathrm{~s}]$ & /PD_300Cu & PD_300Cu_nn.sdt \\
\hline & 100218 & Morales & 1 & 160 & 0.7 & 1.943 & 0 & $5[2.3 \mathrm{~s}]$ & /PD_160Al 1slit & PD_160Al 1slit_nn.sdt \\
\hline & 100218 & Morales & 1 & 160 & 4.35 & 1.943 & 1.905 & $8[2.8 \mathrm{~s}]$ & /PD_160AlCu1slit & PD_160AlCu1slit_nn.sdt \\
\hline
\end{tabular}

\section{Dark current, mid-range, bright field and $I_{o}$}

Root Data Path: /Working/TP35_Industrial_CT_of_HME/LLNL/None/HEAFCAT/None/PD_100218/\{sub directory\}/Raw_Data

\begin{tabular}{|c|c|c|c|c|c|c|c|}
\hline Slits & $\begin{array}{c}\mathbf{k V} \\
\mathbf{p} \\
\end{array}$ & Filter & \{sub directory\} & $\begin{array}{l}\text { Dark Image } \\
\text { File Name } \\
\end{array}$ & $\begin{array}{c}\text { Mid-Brightness Image } \\
\text { File Name } \\
\end{array}$ & $\begin{array}{c}\text { Max Brightness Image } \\
\text { File Name }\end{array}$ & $\begin{array}{c}\text { Io Image } \\
\text { File Name }\end{array}$ \\
\hline 2 & 100 & $\mathrm{Al}$ & /PD_100Al & PD_100Aldrk.sdt & PD_100Almid.sdt & PD_100Allit.sdt & PD_100Albak.sdt \\
\hline 2 & 160 & $\mathrm{Al}$ & /PD_160Al & PD_160Aldrk.sdt & PD_160Almid.sdt & PD_160Allit.sdt & PD_160Albak.sdt \\
\hline 2 & 160 & $\mathrm{AlCu}$ & /PD_160AlCu & PD_160AlCudrk.sdt & PD_160AlCumid.sdt & PD_160AlCulit.sdt & PD_160AlCubak.sdt \\
\hline 2 & 300 & $\mathrm{Cu}$ & /PD_300Cu & PD_300Cudrk.sdt & PD_300Cumid.sdt & PD_300Culit.sdt & PD_300Cubak.sdt \\
\hline 1 & 160 & $\mathrm{Al}$ & /PD_160Al1slit & PD_160Al1slitdrk.sdt & PD_160Al1slitmid.sdt & PD_160Al1slitlit.sdt & PD_160Al1slitbak.sdt \\
\hline 1 & 160 & $\mathrm{AlCu}$ & /PD_160AlCu1slit & PD_160AlCu1slitdrk.sdt & PD_160AlCu1slitmid.sdt & PD_160AlCu1slitlit.sdt & PD_160AlCu1slitbak.sdt \\
\hline
\end{tabular}

\footnotetext{
$1 \mathrm{nn}$ - are the file number for each individual data file
} 


\section{Reconstruction}

Reconstructed by: Travis White

Date: 02/23/2010

Location: LLNL

Computer: Dell Precision 670 and Dell Precision 690

Reconstruction Software

Software: IMGREC

Version: 2.8.1.1c11

\section{Script Files}

LLNL_100Al_2slit-PD_100223.txt

LLNL_160Al_2slit-PD_100223.txt

LLNL_160AlCu_2Slit-PD_100222.txt

LLNL_300Cu_2Slit-PD_100222.txt

LLNL_160Al_1slit-PD_100222.txt

LLNL_160AlCu_1slit-PD_100222.txt

\section{Reconstructed Specimen Files(s)}

Root Data Path: /Working /TP35_Industrial_CT_of_HME/LLNL/None/HEAFCAT/None/PD_100218/\{sub directory\}/

\begin{tabular}{|c|c|c||c||c|}
\hline Slits & $\mathbf{k V p}$ & Filter & \{sub directory\} & Reconstruction file name \\
\hline \hline 2 & 100 & $\mathrm{Al}$ & $/ \mathrm{PD} \_100 \mathrm{Al}$ & recobj_nn ${ }^{1}$.sdt \\
\hline 2 & 160 & $\mathrm{Al}$ & /PD_160Al & recobj_nn.sdt \\
\hline 2 & 160 & $\mathrm{AlCu}$ & /PD_160AlCu & recobj_nn.sdt \\
\hline 2 & 300 & $\mathrm{Cu}$ & /PD_300Cu & recobj_nn.sdt \\
\hline 1 & 160 & $\mathrm{Al}$ & /PD_160Al1slit & recry_nn.sdt,ry_nn.sdt \\
\hline 1 & 160 & $\mathrm{AlCu}$ & /PD_160AlCu1slit & recry_nn.sdt,ry_nn.sdt \\
\hline
\end{tabular}

\section{Observations:}

\footnotetext{
${ }^{1} \mathrm{nn}$ - are the file number for each individual data file
} 


\section{REFERENCES}

1. "Standard Operating Procedure - Industrial Computed Tomography System Data Collection of Home-Made Explosives,” U.S. Department of Homeland Security Science and Technology Directorate, DHS/STD/TSL-xx-xx, July 9, 2009.

2. Jerel A. Smith, Daniel J. Schneberk, Jeffrey S. Kallman, Harry E. Martz, Jr., David Hoey, Documentation of the LLNL and Tyndall Micro-Computed-Tomography Systems, Version 091216, Lawrence Livermore National Laboratory, LLNL-TR-421377, December 17, 2009.

3. Harry E. Martz, Jr., and Carl Crawford, Validation of Explosive Simulants Requirement Specification, Version 12, Lawrence Livermore National Laboratory, LLNL-TR-416983-REV 1, October 26, 2009.

4. Maurice B. Aufderheide and W. Travis White III, HADES Calculations of the X-ray Linear Absorption Coefficient and Effective Atomic Number in Selected Materials, Lawrence Livermore National Laboratory, LLNL-TR-xxxx-DRAFT, February 12, 2010. 